\title{
Myeloid-derived suppressor cells are increased and correlated with type 2 immune responses, malnutrition, inflammation, and poor prognosis in patients with breast cancer
}

\author{
KENJI GONDA ${ }^{1}$, MASAHIKO SHIBATA ${ }^{2,3}$, TOHRU OHTAKE ${ }^{4}$, \\ YOSHIKO MATSUMOTO ${ }^{4}$, KAZUNOSHIN TACHIBANA ${ }^{4}$, NORIKO ABE ${ }^{4}$, \\ HITOSHI OHTO ${ }^{5}$, KENICHI SAKURAI ${ }^{6}$ and SEIICHI TAKENOSHITA ${ }^{4}$
}

\begin{abstract}
${ }^{1}$ Department of Surgery, Japan Community Healthcare Organization Nihonmatsu Hospital, Nihonmatsu, Fukushima 964-8501;
${ }^{2}$ Department of Gastroenterological Oncology, Comprehensive Cancer Center, Saitama Medical University International Medical Center, Hidaka, Saitama 350-1298; Departments of ${ }^{3}$ Tumor and Host Bioscience,

${ }^{4}$ Organ Regulatory Surgery and ${ }^{5}$ Blood Transfusion and Transplantation Immunology, Fukushima Medical University, Fukushima 960-1295; ${ }^{6}$ Division of Breast and Endocrine Surgery,

Department of Surgery, Nihon University School of Medicine, Tokyo 173-8610, Japan
\end{abstract}

Received July 2, 2016; Accepted February 23, 2017

DOI: $10.3892 / \mathrm{ol} .2017 .6305$

\begin{abstract}
Myeloid-derived suppressor cells (MDSCs) have been identified in the majority of patients and experimental mice with tumors by their suppression of $\mathrm{T}$ cell activation. MDSCs have also been reported to be associated with chronic inflammation. In advanced cancer, the T helper (Th) cell balance tends to shift from Th1 to Th2 predominance, and immune function, including cell-mediated immunity, is impaired by cytokines produced by Th 2 cells. The present study examined the correlations between MDSC levels and inflammation, immune suppression, malnutrition, and poor prognosis in 155 patients with breast cancer. The levels of MDSCs in preoperative patients and in patients with recurrent breast cancer were significantly higher compared with postoperative patients, patients with recurrent breast cancer who received chemotherapy and healthy volunteers. The MDSC levels of preoperative patients were significantly positively correlated with interleukin (IL)-6 production by peripheral blood mononuclear cells (PBMCs), the neutrophil/lymphocyte ratio and C-reactive protein, and were negatively correlated with the production of interferon- $\gamma$ and IL-12, serum concentration of rapid turnover protein, and the stimulation index. These patients were divided into two groups based on the levels
\end{abstract}

Correspondence to: Dr Kenji Gonda, Department of Surgery, Japan Community Health Care Organization Nihonmatsu Hospital, 1-553 Naritamachi, Nihonmatsu, Fukushima 964-8501, Japan E-mail: gonken@lime.plala.or.jp

Key words: Myeloid-derived suppressor cells, immune suppression, breast cancer, inflammation, Th1/Th2 balance, malnutrition, five-year survival rate of MDSCs. In preoperative patients with MDSC levels $>1.0 \%$ of total PBMCs, the overall survival of patients with stage IV disease was significantly shorter compared with other disease stages, and was also significantly shorter compared with patients with MDSC levels $<1.0 \%$ of total PBMCs. Thus, the MDSC levels of preoperative patients may function as a good prognostic indicator, particularly in patients with advanced breast cancer.

\section{Introduction}

Malignant breast tumors represent one of the most commonly occurring cancers in women, and are a leading cause of cancer-associated mortality (1). While advances in surgery, radiotherapy, chemotherapy and hormone replacement therapy have irrefutably enhanced early diagnosis and treatment, no effective therapy exists for advanced, invasive, metastatic breast cancer (2). Strong evidence for the effectiveness of immunotherapy has been provided in previous reports, and this approach is expected to be effective in such cases (3). However, the majority of cancer immunotherapies that have been developed in experimental animal models and have been tested in clinical trials have not been effective, although certain treatments have demonstrated modest clinical effects (4-7). Previous studies have identified cells of myeloid origin that suppress tumor immunity, making them an impediment to cancer immunotherapy. These suppressive myeloid cells were initially described in patients with cancer six decades ago $(8,9)$, and the importance of their function in the immune system has begun to be appreciated $(10,11)$. Accumulating evidence has demonstrated that myeloid-derived suppressor cells (MDSCs), a population of cells with suppressive activity, contribute to the negative regulation of immune responses that occurs in diseases including cancer. MDSCs in mice are characterized by the expression of cell surface molecules 
that are detected by antibodies targeting Gr1 and cluster of differentiation (CD)11b $(12,13)$. MDSC phenotype variation is consistent with the idea that these cells belong to a diverse family of cells in various, intermediate stages of myeloid cell differentiation (12). In humans, MDSCs are defined as CD14, CD11b cells or, more specifically, as cells expressing the common myeloid marker CD33 but lacking markers of mature myeloid and lymphoid cells and the MHC class II molecule HLA-DR (14). In the present study, CD14-, CD11b+ and CD33+ cells were considered to be MDSCs.

In 1986, Mosmann et al (15) described the existence of two types of $\mathrm{T}$ helper (Th) populations; Th1 and Th2. Following T-cell receptor ligation, Th0 cells differentiate into specific subsets characterized by their functions and cytokine production profiles. Th1 lymphocytes are induced by interleukin (IL) -2 , tend to produce IL-12 and interferon (IFN)- $\gamma$, and are involved in cellular immunity. Th2 lymphocytes primarily induce humoral immunity and produce IL-4, IL-6 and IL-10. The immune function of patients with cancer, in particular cell-mediated immunity, is known to be impaired and the Th cell balance during this disease tends to shift from Th1 to Th2 predominance. In a previous study concerning gastric and colorectal cancer, the production of IL-12 decreased as the diseases progressed and production of IFN- $\gamma$ decreased uniformly in patients with cancer compared with healthy volunteers (16).

At present, there is a paucity of information concerning MDSCs in patients with breast cancer, in particular regarding the relationship of MDSCs with immune status (17). The present study detected MDSCs in circulating blood from patients with breast cancer and these levels were analyzed. In addition, MDSCs were also detected in the pleural effusion of a patient with metastatic breast cancer. Finally, the effects of chemotherapy on MDSCs were addressed.

\section{Materials and methods}

Study subjects. In the present study, a total of 155 patients were enrolled. Patients were aged between 40 and 84 years (median, 58.0 years) and had histologically confirmed breast cancer that was treated in the Department of Organ Regulatory Surgery, Fukushima Medical University (Fukushima, Japan) between January 2011 and June 2016. A total of 110 preoperative patients, including 18 patients with stage I disease, 39 with stage II, 17 with stage III, and 36 with stage IV cancer; 23 postoperative patients, including 5 with stage I, 10 with stage II, 7 with stage III, and 1 with stage IV cancer in accordance with National Comprehensive Cancer Network (NCCN) Clinical Practice Guidelines in Oncology: Breast Cancer Screening and Diagnosis (18); 22 recurrent patients that had received a regimen of chemotherapy (6 cycles of $1,000 \mathrm{mg} / \mathrm{m}^{2}$ gemacitabine on days 1 and 8 for 21 days); and 18 healthy volunteers were enrolled. None of the preoperative patients had received any anticancer treatment. A total of 177 blood samples were collected from the 155 patients with breast cancer, including double sampling in recurrent patients (preand post-chemotherapy), and from the 18 healthy volunteers. Peripheral blood mononuclear cells (PBMCs) were isolated using the Ficoll density gradient centrifugation method (Ficoll-Hypaque; GE Healthcare Life Sciences, Chalfont, UK) from $20 \mathrm{ml}$ venous blood collected in EDTA tubes. Aliquots of PBMCs were cryopreserved in freezing medium (BLC-1; Juji-Field Co. Ltd, Tokyo, Japan). The plasma was separated by centrifugation at $1,500 \mathrm{x}$ g for $10 \mathrm{~min}$ in a refrigerated centrifuge at $4^{\circ} \mathrm{C}$ and kept at $-80^{\circ} \mathrm{C}$ until analysis. Double samples (pre- and post-chemotherapy) from pleural effusions were examined. A total of $1 \times 10^{6}$ cells from $100-\mathrm{ml}$ plural effusion samples were isolated by centrifugation at $400 \mathrm{x}$ g for $30 \mathrm{~min}$ at $4^{\circ} \mathrm{C}$ using the Ficoll density gradient centrifugation method, and subjected to flow cytometric analysis as described. The present study was approved by the Ethics Committee of Fukushima Medical University (2011-2016). Written informed consent was obtained from all enrolled patients and healthy donors.

Flow cytometric analysis. A total of $1 \times 10^{6}$ frozen PBMCs were thawed rapidly and washed three times with PBS. A three-color flow cytometric analysis was performed with a cocktail of antibodies, consisting of fluorescent isothiocyanate (FITC)-conjugated anti-CD14 (cat. no. ab28061; $20 \mu \mathrm{l}$; Abcam, Cambridge, UK), phycoerythrin (PE)-conjugated anti-CD11b (cat.no. IM-2581 U; dilution, 0.2 mg/ml; Beckman Coulter, Inc., Brea, CA, USA), and phycoerythrin cyanin (PC)5.1-conjugated anti-CD33 (cat. no. FAB1137F; $20 \mu \mathrm{l}$; Beckman Coulter, Inc.). Following incubation with PBS for $30 \mathrm{~min}$ at $4{ }^{\circ} \mathrm{C}$, samples were washed with fluorescence-activated cell sorting (FACS) buffer. Pellets were subsequently resuspended in $800 \mu$ FACS buffer. Data acquisition and analysis were performed on a FACS Aria II flow cytometer (BD Biosciences, San Jose, CA, USA; Fig. 1) using Flow Jo v10.2 software (Tree Star, Inc., Ashland, OR, USA). The labeled cells were first gated (R1) based on their expression of CD14; R1 was composed of CD14- cells. The fraction of cells in this population that expressed the myeloid markers CD11b and CD33 was then determined. Therefore, in the present study, MDSCs were defined as CD14, ${ }^{-} \mathrm{CD}_{11 b^{+} \text {and }}$ $\mathrm{CD}_{3}{ }^{+}$cells. The percentage of MDSCs was calculated as a percentage of the total PBMCs.

Cytokine production assay. To assess cytokine production, $1 \times 10^{6}$ PBMCs were cultured in $1 \mathrm{ml}$ RPMI-1640 medium (Wako Pure Chemical Industries, Ltd., Osaka, Japan) containing $10 \%$ heat-inactivated fetal calf serum (FCS; Gibco; Thermo Fisher Scientific, Inc., Waltham, MA, USA) and $100 \mu \mathrm{g} / \mathrm{ml}$ phytohemagglutinin (PHA; Sigma-Aldrich; Merck $\mathrm{KGaA}$, Darmstadt, Germany) for $24 \mathrm{~h}$ in $5 \% \mathrm{CO}_{2}$ at $37^{\circ} \mathrm{C}$. Subsequently, 1x10 ${ }^{6}$ PBMCs were seeded into a 96-well plate (Costar, Lowell, MA, USA) in the presence of PHA. Following cultivation, IL- 6 and IFN- $\gamma$ in supernatants were measured using a Quantikine ELISA kit (Human IL-6 immunoassay, cat. no. D6050; Human IFN- $\gamma$ immunoassay, cat. no. DIF50), according to the manufacturer's protocol (R\&D Systems, Inc., Minneapolis, MN, USA).

At the same time, $1 \times 10^{6} \mathrm{PBMCs}$ were cultured in $1 \mathrm{ml}$ RPMI-1640 medium containing 10\% heat-inactivated FCS and $0.075 \% / \mathrm{vol}$ of Staphylococcus aureus Cowan1 (SAC; Sigma-Aldrich; Merck $\mathrm{KGaA}$ ) for $24 \mathrm{~h}$ in $5 \% \mathrm{CO}_{2}$ at $37^{\circ} \mathrm{C}$. PBMCs $\left(1 \times 10^{6}\right)$ were then seeded into a 96 -well plate in the presence of SAC. Following cultivation, IL-12 in supernatant was measured using a Quantikine ELISA kit (Human IL-12 immunoassay, cat. no. DP400) according to the manufacturer's 
protocol (R\&D Systems, Inc.). Absorbance at $450 \mathrm{~nm}$ was measured using an ELISA micro plate reader.

Lymphocyte proliferation assay. Lymphocyte proliferation assays were performed using A total of $1 \times 10^{6}$ cells $/ \mathrm{ml}$ were suspended in RPMI-1640 medium containing 20\% fetal bovine serum (FBS; Kojin Bio Co., Ltd., Saitama, Japan). Following the addition of $100 \mu \mathrm{g} / \mathrm{ml}$ PHA into the PBMC culture wells, the culture was kept at $37^{\circ} \mathrm{C}$ in a $5 \% \mathrm{CO}_{2}$ atmosphere and PHA mitogenesis was observed 3 times for $80 \mathrm{~h}$ by a light microscope (magnification, $\mathrm{x} 10$ ). A total of $10 \mu \mathrm{l}^{3} \mathrm{H}$-thymidine (Japan Radioisotope Association, Tokyo, Japan) was added to the wells (18.5 Beq/well) for the last $72 \mathrm{~h}$ of incubation. Cells were harvested using an auto cell harvester with a UniFilter Plate (Bio Tec Co., Ltd. Tokyo, Japan) and ${ }^{3} \mathrm{H}$-thymidine incorporation was determined using a liquid scintillation counter (PerkinElmer, Inc., Waltham, MA, USA) and was expressed as counts per minute (cpm). The stimulation index (SI) was obtained by calculating total $\mathrm{cpm} / \mathrm{control} \mathrm{cpm}$. The controls were PBMCs that had not been subjected to PHA addition.

Statistical analysis. All statistical analyses were performed using Excel Statics 2012 software for Windows (Social Survey Research Information Co., Ltd. Tokyo, Japan). The differences between groups were determined using Student's t-tests. Relationships between two variables were quantified using Spearman's rank correlation coefficient tests. All data are presented as the mean \pm standard deviation. $\mathrm{P}<0.05$ was considered to indicate a statistically significant difference. When measuring overall survival (OS), follow-up data that were not reached as of the last follow-up date or at 1,500 days were censored. The prognoses of the patients were analyzed using Kaplan-Meier method curves and the log-rank test was used to determine the univariate significance of the variables. In 110 patients with preoperative breast cancer, multivariate Cox regression analysis of survival was performed according to MDSC levels, tumor size, molecular subtype of the tumor, Ki-67 status, lymph node status, and histological grading in accordance with NCCN Clinical Practice Guidelines in Oncology (18). A Cox proportional-hazards model was used to simultaneously examine the effects of multiple covariates on survival. The effect of each individual variable was described by the hazard ratio, with a $95 \%$ confidence interval.

\section{Results}

MDSC levels in pre- and post-operative patients, in patients with recurrent breast cancer, and in patients with recurrent breast cancer following chemotherapy. MDSCs were successfully detected by flow cytometry. Representative flow cytometric data was obtained for normal healthy volunteers (Fig. 1A), preoperative patients (Fig. 1B), postoperative patients (Fig. 1C), patients with recurrent breast cancer (Fig. 1D), and patients receiving gemcitabine (Fig. 1E). The percentage of MDSCs in the PBMCs of 177 samples from 155 patients with breast cancer and from 18 healthy volunteers was analyzed. The percentage of MDSCs in the PBMCs from 110 preoperative patients with breast cancer was significantly increased compared with the 18 healthy volunteers $(2.27 \pm 1.39$ and $1.11 \pm 0.54 \%$, respectively; $\mathrm{P}<0.05$; Fig. 2). Of the 110 preoperative patients, the MDSC levels of patients with stage I, stage II, stage III, and stage IV diseases were $1.94 \pm 1.23$, $2.91 \pm 0.35,1.26 \pm 0.41$ and $2.98 \pm 0.40 \%$, respectively, and those of stage I, stage II, and stage IV were significantly higher than the levels of healthy volunteers (Fig. 2). The MDSC levels of 23 postoperative patients, 22 recurrent patients, and 22 recurrent patients who received chemotherapy were $1.31 \pm 0.31$, $1.91 \pm 2.08$, and $1.21 \pm 0.52 \%$, respectively (Fig. 2). The MDSC levels were also significantly higher in recurrent patients prior to chemotherapy treatment compared with in healthy volunteers ( $\mathrm{P}<0.05$; Fig. 2). The MDSC levels of postoperative patients were significantly lower than those of preoperative patients $(\mathrm{P}<0.05$; Fig. 2$)$ and were equivalent to the range in healthy volunteers. The MDSC levels of the patients with recurrent breast cancer following chemotherapy were lower than those in patients with recurrent breast cancer prior to chemotherapy and were not significantly different from the levels in healthy volunteers. In summary, the MDSC levels were increased in preoperative patients, decreased following removal of the tumor, and increased again with recurrence. In addition, MDSC levels of patients with recurrent breast cancer decreased following chemotherapy (Fig. 2).

MDSC levels were correlated with Th2 polarization, malnutrition, inflammation and immune suppression. The levels of MDSCs in preoperative patients were correlated with immune suppression, in particular Th2 polarization, malnutrition and inflammation. Regarding Th2 polarization, the MDSC levels of preoperative patients were significantly positively correlated with IL- 6 production $(\mathrm{r}=0.49, \mathrm{P}<0.01$; Fig. $3 \mathrm{~A})$ and were significantly inversely correlated with IFN $-\gamma(\mathrm{r}=-0.49, \mathrm{P}<0.01$; Fig. 3B) and IL-12 ( $\mathrm{r}=-0.46, \mathrm{P}<0.01$; Fig. $3 \mathrm{C})$ production. Regarding malnutrition, the MDSCs levels of preoperative patients were significantly negatively correlated with the short turnover protein: retinol binding protein $(\mathrm{r}=-0.53, \mathrm{P}<0.01$; Fig. 4A), prealbumin ( $r=-0.59, \mathrm{P}<0.01$; Fig. $4 \mathrm{~B})$, and transferrin $(\mathrm{r}=-0.52, \mathrm{P}<0.01$; Fig. 4C). In terms of inflammation and the SI, the MDSC levels of preoperative patients were significantly positively correlated with the neutrophil/lymphocyte ratio (NLR; r=0.54, P<0.01; Fig. 5A) and C-reactive protein (CRP; $\mathrm{r}=0.59, \mathrm{P}<0.01$; Fig. 5B) and were significantly negatively correlated with the SI ( $r=-0.50, \mathrm{P}<0.01$; Fig. $5 \mathrm{C})$.

Effect of MDSC levels in patients with stages I, II, III, and IV breast cancer on overall survival. The preoperative patients were separated into 2 groups based on whether their circulating MDSC levels were higher or lower than $1.0 \%$ of total PBMCs. The OS of these 2 groups was then compared. In patients with MDSC levels $>1.0 \%$, the OS of stage IV disease was significantly shorter than that of stage I, II, or III disease $(\mathrm{P}<0.01$; Fig. 6A), and no difference in survival was observed between stages I, II, and III. On the other hand, there were no differences in OS between stage I-IV breast cancer patients with MDSC levels $<1.0 \%$ (Fig. 6B). The OS of stage IV patients with MDSC levels that were $>1.0 \%$ was significantly shorter than that of patients with MDSC levels $<1.0 \%$ ( $\mathrm{P}<0.05$; Fig. 7).

MDSCs and chemotherapy. It has previously been reported that gemcitabine treatment of tumor-bearing mice significantly inhibited tumor growth, reduced splenomegaly, 


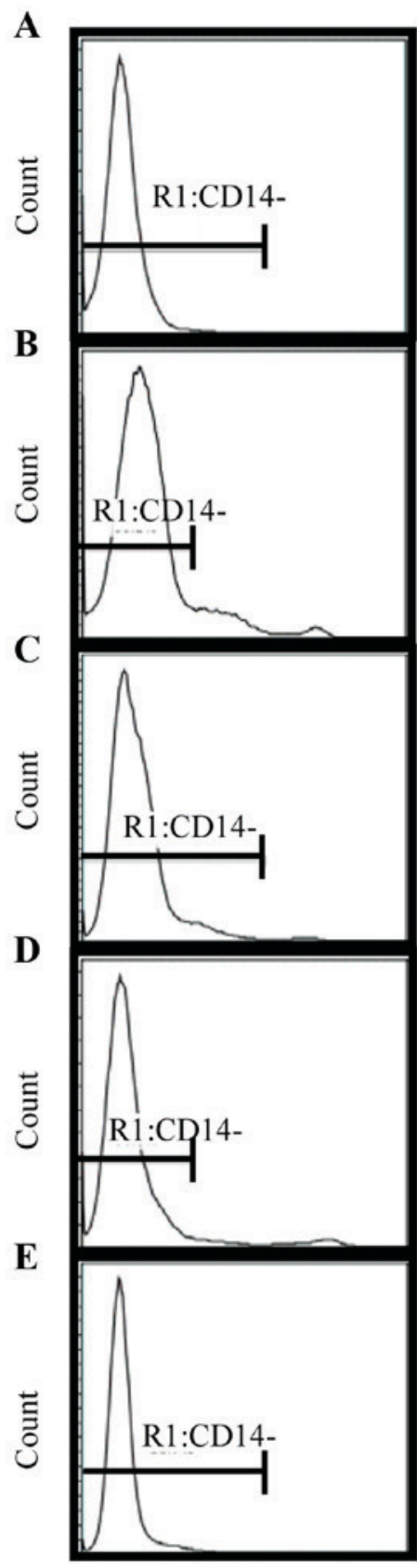

$\mathrm{CD} 14$

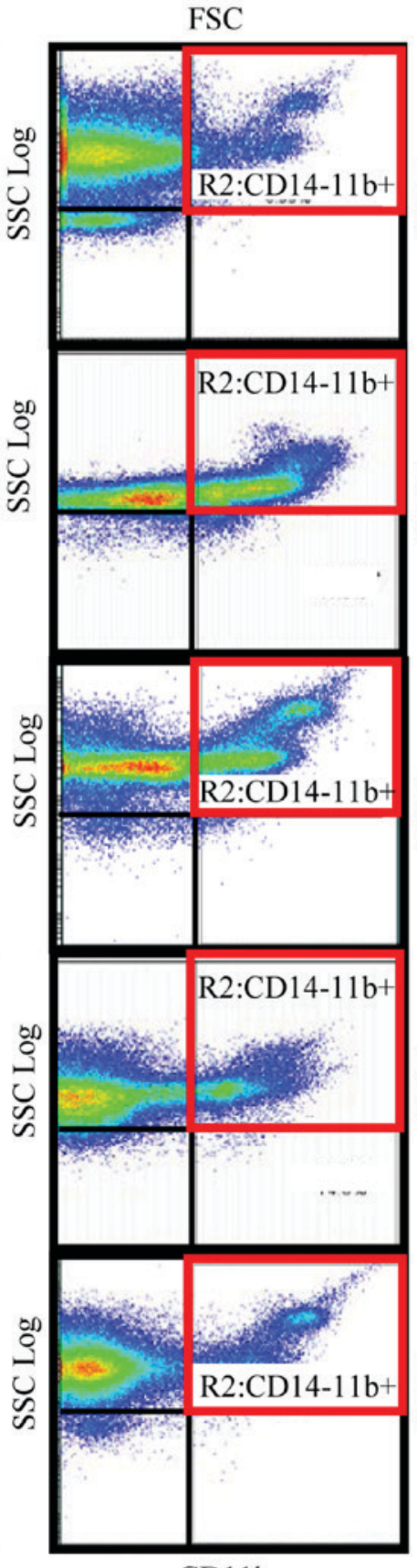

CD11b

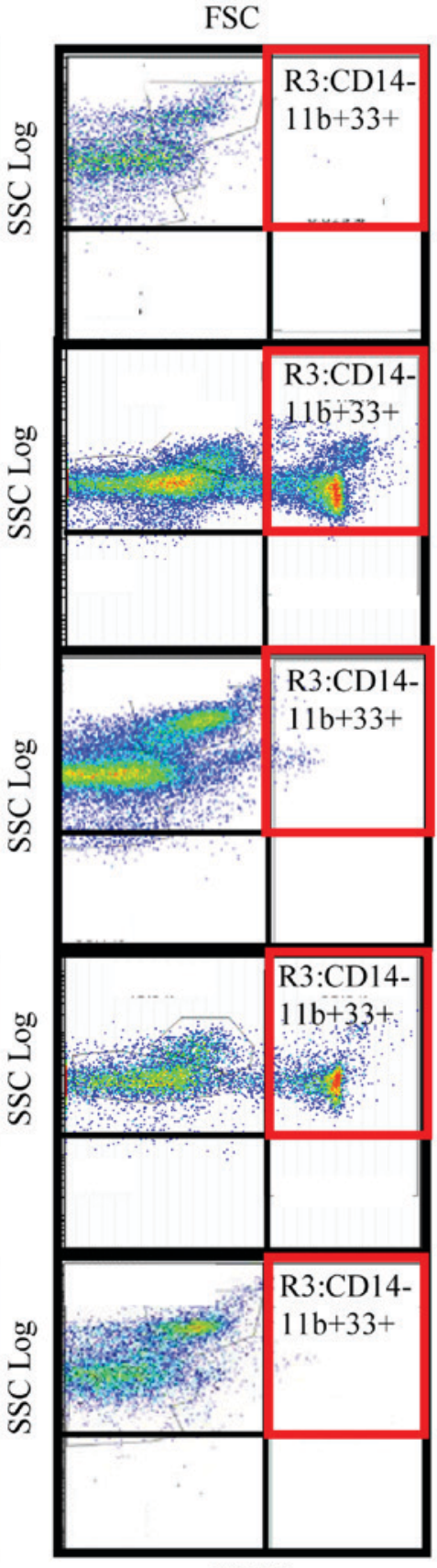

$\mathrm{CD} 33$

Figure 1. Immunophenotyping of MDSCs, using flow cytometry. R1 was a forward scatter/side scatter plot to gate out cell debris and a CD14/side scatter plot to exclude CD14 positive events. R1 was composed of CD14 cells. This population was then gated by a CD11b/side scatter plot to give the R2 cell fraction, and the R3 fraction of the cells was then gated by a CD33/side scatter plot. Therefore, R3 was defined as MDSCs which were gated by CD14, CD11b and CD33 expression. The MDSC percentage was calculated as the percentage of total nucleated cells in the whole blood sample. Representative flow cytometry results are shown for (A) a healthy volunteer; (B) a preoperative patient with stage IV breast cancer; (C) a postoperative patient; (D) a patient with recurrent breast cancer; and (E), a patient with recurrent breast cancer receiving gemcitabine chemotherapy. MDSC, myeloid-derived suppressor cell; R1, initial gating; CD, cluster of differentiation; FSC, forward scattered light.

and significantly decreased the proportion of MDSCs in the spleen (19). Patients with recurrent breast cancer in the present study had received 6 cycles of gemcitabine $\left(1,000 \mathrm{mg} / \mathrm{m}^{2}\right)$. It was therefore determined whether gemcitabine therapy had altered MDSC levels in these patients. MDSC levels were analyzed in these patients prior to chemotherapy and 14 days following the last cycle of chemotherapy. There was a significant decrease in MDSC levels following chemotherapy compared with those prior to chemotherapy $(1.91 \pm 1.50$ and $1.18 \pm 0.71 \%$, respectively; $\mathrm{P}=0.01$; Fig. 8).

MDSCs in pleural effusion and the effect of chemotherapy. A 69-year-old female who had undergone mastectomy and axillary dissection two years previously was revealed to have new lung metastasis and pleural effusion. MDSCs were detected in the pleural effusion using the same experimental procedure as that described above for PBMCs. The effect of 


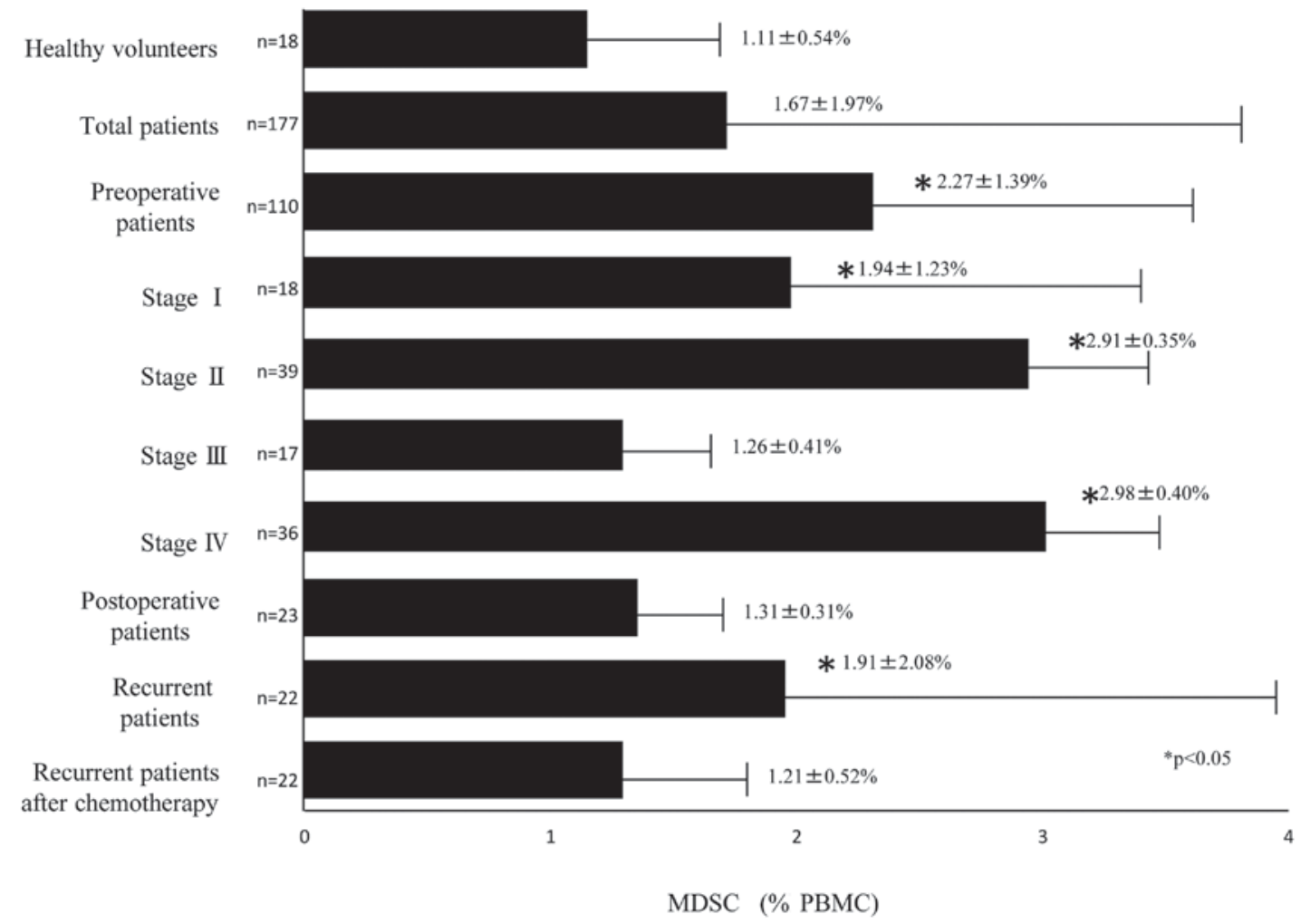

Figure 2. Percentage of circulating MDSCs in patients with breast cancer and in healthy volunteers. ${ }^{*} \mathrm{P}<0.05$ vs. healthy volunteers. MDSC, myeloid-derived suppressor cell; PBMC, peripheral blood mononuclear cells.

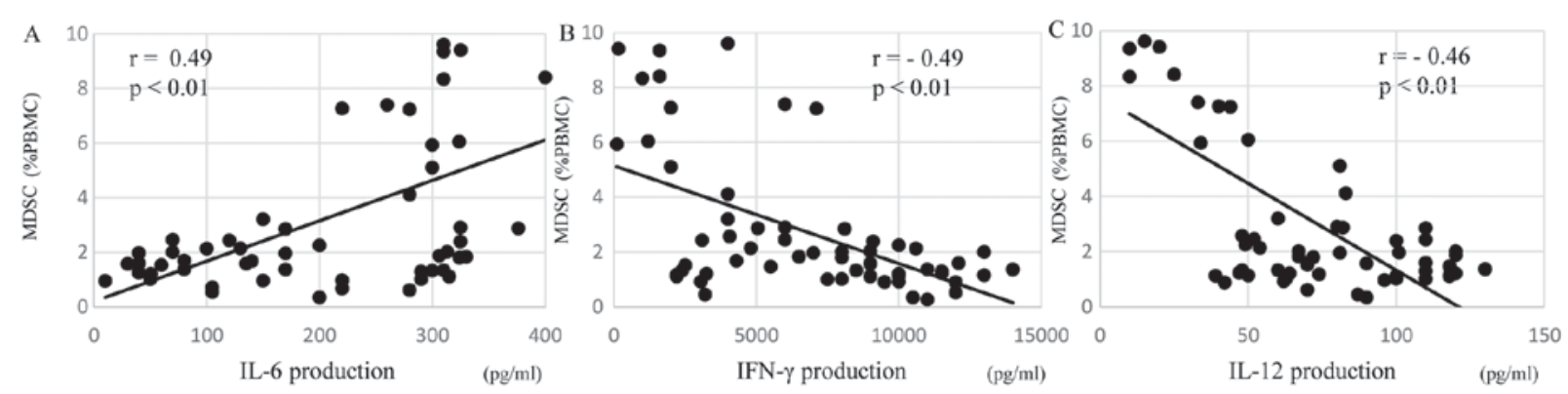

Figure 3. MDSC levels and cytokine production. In preoperative patients, MDSC levels were significantly positively correlated with (A) IL-6 production, and were significantly negatively correlated with (B) IFN- $\gamma$ and (C) IL-12 production. MDSC, myeloid-derived suppressor cell; IL, interleukin; IFN- $\gamma$, interferon- $\gamma$; PBMC, peripheral blood mononuclear cells.
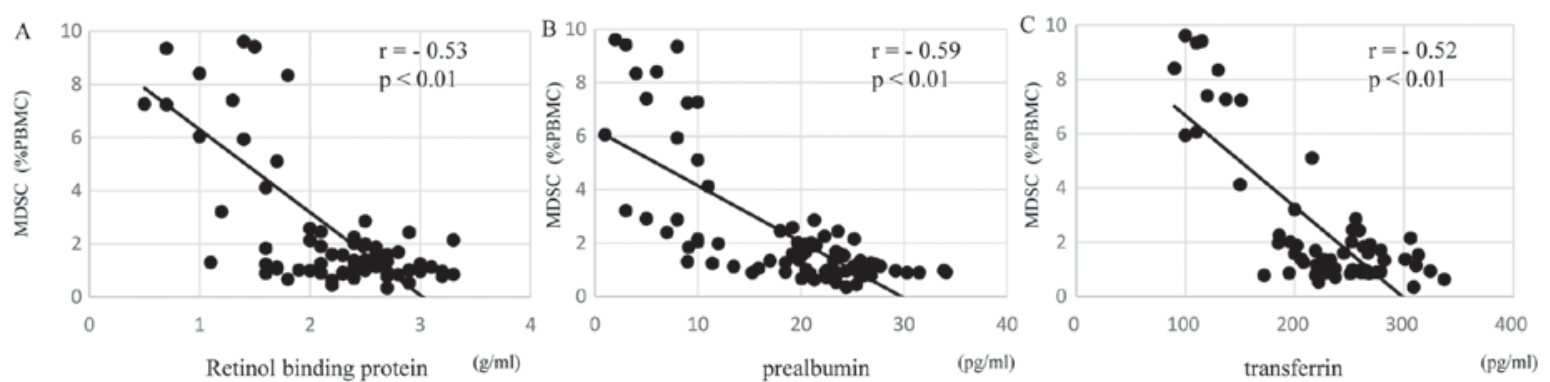

Figure 4. MDSC levels and malnutrition. In preoperative patients, MDSC levels were significantly negatively correlated with (A) retinol binding protein, (B) prealbumin and (C) transferrin. MDSC, myeloid-derived suppressor cell; PBMC, peripheral blood mononuclear cells.

chemotherapy on MDSC levels was then examined in the pleural effusion of this patient. MDSC levels in the pleural effusion were decreased from $34.5 \%$ prior to chemotherapy (Fig. 9A) to $11.0 \%$ following 6 cycles of gemcitabine treatment 

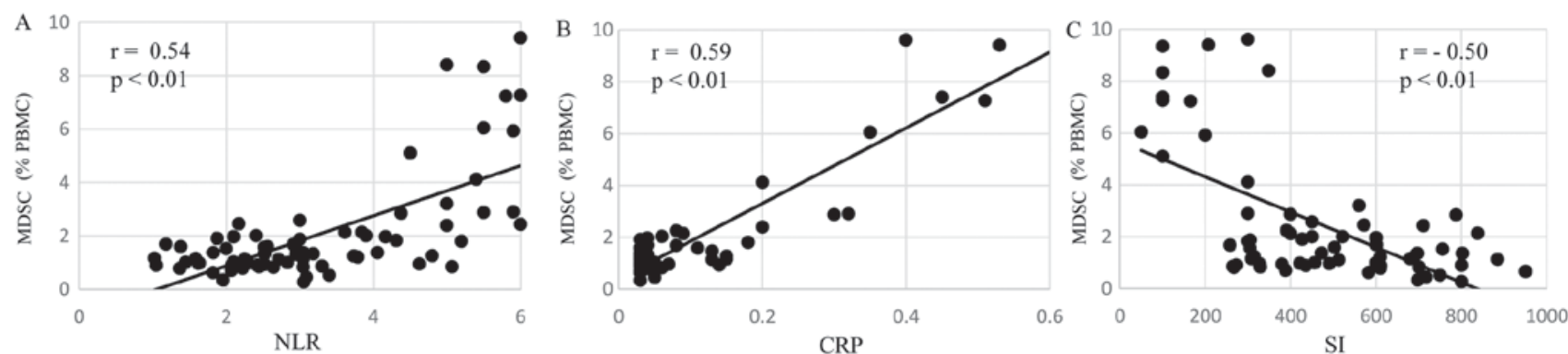

Figure 5. MDSC levels, inflammation and the SI. In preoperative patients, MDSC levels were significantly positively correlated with (A) the NLR and (B) CRP, and were significantly negatively correlated with (C) the SI. MDSC, myeloid-derived suppressor cell; SI, stimulation index; NLR, neutrophil/lymphocyte ration; CRP, C-reactive protein; PBMC, peripheral blood mononuclear cells.

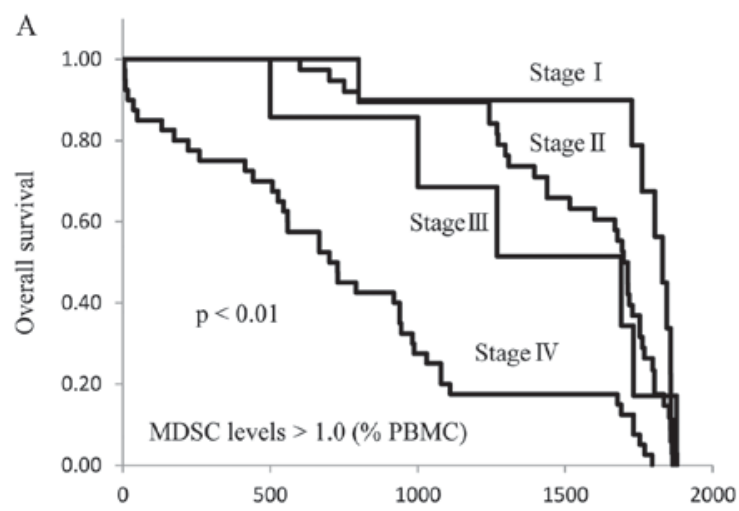

Time (days)

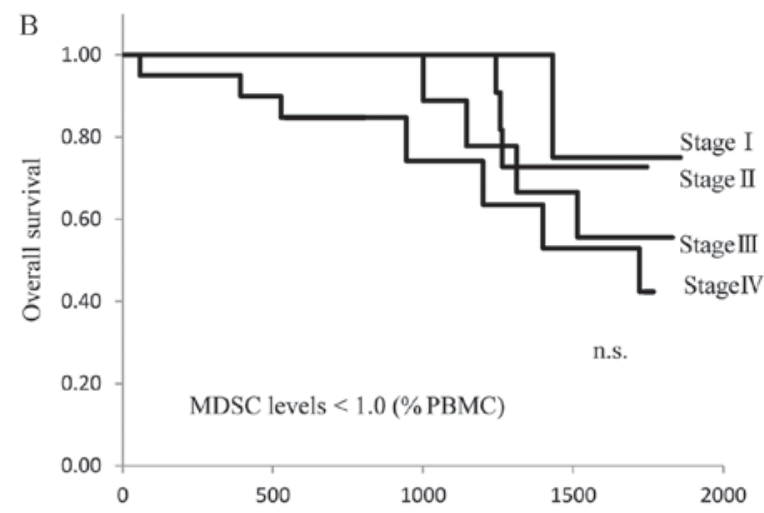

Time (days)

Figure 6. MDSC levels of preoperative patients and overall survival. Kaplan-Meier analyses for overall survival according to MDSC levels (A) $>1.0 \%$ and (B) $<1.0 \%$. Patients were stratified into four groups (stage I, II, III and IV). Survival analysis was performed in all preoperative patients with follow-up. MDSC, myeloid-derived suppressor cell; PBMC, peripheral blood mononuclear cells.

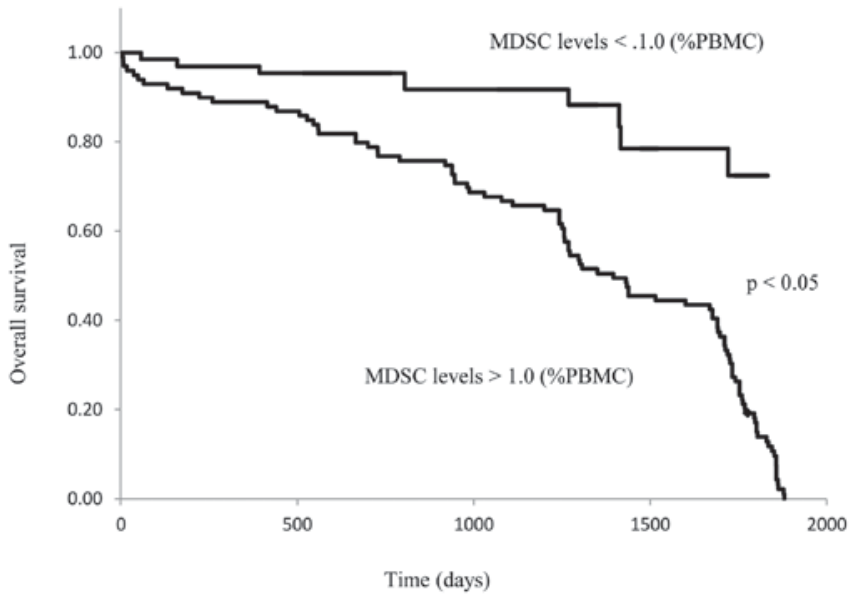

Figure 7. MDSC levels of patients with stage IV breast cancer and overall survival. Kaplan-Meier analysis for overall survival according to MDSC levels. The patients were stratified into two groups: MDSCs $<1.0 \%$ and MDSCs $>1.0 \%$. Survival analysis was performed in 36 stage IV patients with follow-up. MDSC, myeloid-derived suppressor cell; PBMC, peripheral blood mononuclear cells.

(Fig. 9B). The MDSC levels in the peripheral blood were also decreased following chemotherapy in this case. Thus, the level of MDSCs in the pleural effusion and in the PBMCs decreased in parallel in response to chemotherapy.

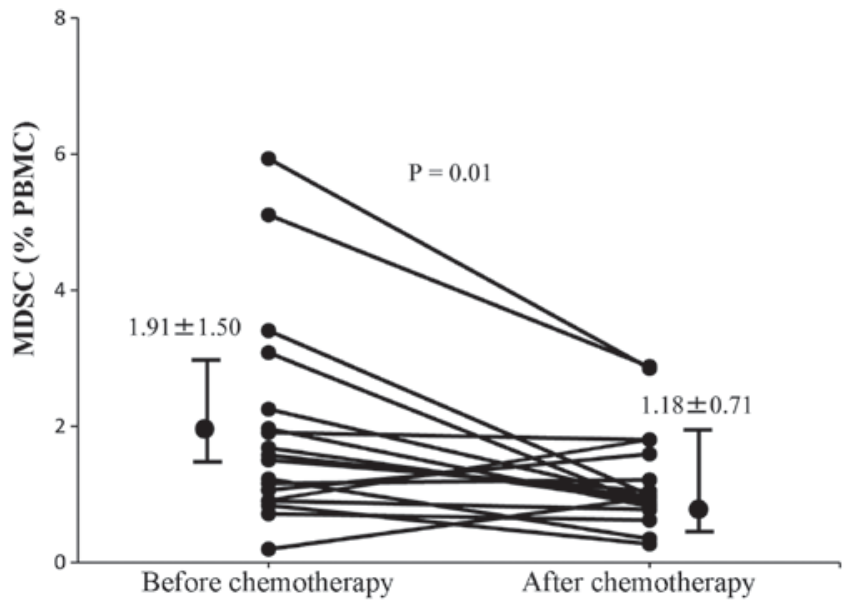

Figure 8. MDSC levels of patients with recurrent breast cancer prior to and following chemotherapy. The MDSC levels of each patient prior to and following chemotherapy are depicted using black circles, and the levels of the same patient are connected using lines. MDSC levels following chemotherapy were significantly decreased compared with those prior to chemotherapy. MDSC, myeloid-derived suppressor cell; PBMC, peripheral blood mononuclear cells

Multivariate regression analysis of survival according to the serum MDSC levels in molecular subtypes of tumors. The present study then examined whether the prognostic value of 
Table I. Multivariate Cox regression analysis of survival according to MDSC levels, tumor size, tumor molecular subtype, Ki-67 status, lymph node status and histological grade.

\begin{tabular}{|c|c|c|c|c|}
\hline Clinicopathological characteristic & Number of patients $(n=110)$ & HR & $95 \% \mathrm{CI}$ & P-value \\
\hline \multicolumn{5}{|l|}{ MDSCs (\% of PBMCs) } \\
\hline$<1$ & 60 & & & \\
\hline$\geq 1$ & 50 & - & - & $<0.05$ \\
\hline \multicolumn{5}{|l|}{ Tumor size (mm) } \\
\hline$<30$ & 46 & - & - & - \\
\hline$\geq 30$ & 64 & 1.05 & $0.77-1.21$ & $<0.10$ \\
\hline \multicolumn{5}{|l|}{ Molecular subtype } \\
\hline Luminal A & 41 & - & - & - \\
\hline Luminal B & 27 & 1.26 & $1.14-1.61$ & $<0.10$ \\
\hline HER2 & 15 & 1.14 & $0.95-1.39$ & $<0.10$ \\
\hline TNBC & 17 & 1.25 & $1.11-1.58$ & $<0.10$ \\
\hline \multicolumn{5}{|l|}{ Ki-67 (\%) } \\
\hline$<50$ & 32 & - & - & - \\
\hline$\geq 50$ & 78 & 1.18 & $0.97-1.40$ & $<0.10$ \\
\hline \multicolumn{5}{|l|}{ Lymph node status } \\
\hline No & 52 & - & - & - \\
\hline$\geq \mathrm{N} 1$ & 58 & 1.29 & $1.12-1.64$ & $<0.10$ \\
\hline \multicolumn{5}{|l|}{ Histological grade } \\
\hline G1 & 27 & - & - & - \\
\hline$\geq \mathrm{G} 2$ & 83 & 1.21 & $1.12-1.53$ & $<0.10$ \\
\hline
\end{tabular}

MDSC, myeloid-derived suppressor cell; PBMC, peripheral blood mononuclear cells; HER2, human epidermal growth factor receptor 2; TNBC, triple-negative breast cancer; HR, hazard ratio; CI, confidence interval.

A

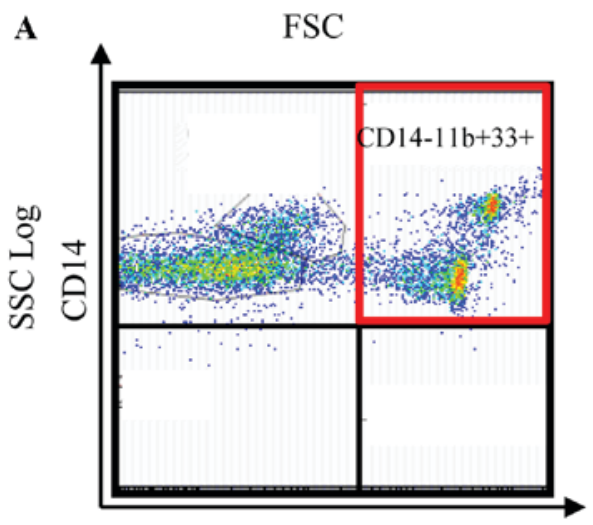

CD11bCD33
B

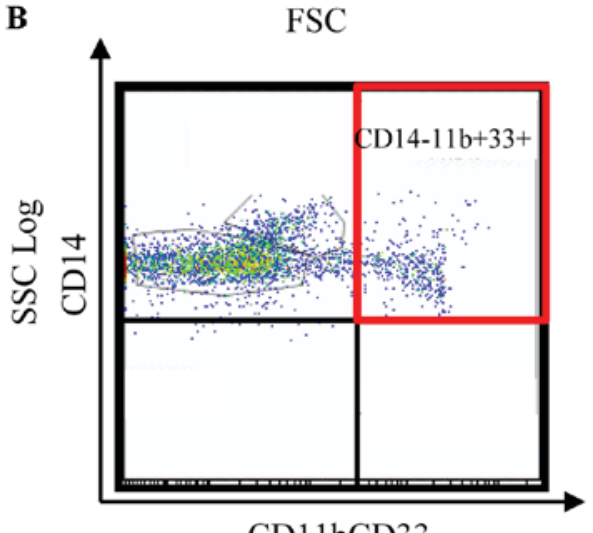

Figure 9. Myeloid-derived suppressor cell levels in pleural effusion (A) prior to and (B) following six cycles of gemcitabine-based chemotherapy. FSC, forward scattered light; CD, cluster of differentiation.

an MDSC level of $>1.0 \%$ remained statistically significant in a multivariate analysis in the total cohort. A multivariate Cox regression model, which included a level of MDSCs $<1.0 \%$, tumor size, molecular subtype of the tumor, including Luminal A, Luminal B, human epidermal growth factor receptor 2 or triple negative breast cancer, Ki-67 status, lymph node status and histological grade was applied. Apart from an MDSC level of $<1.0 \%$, none of the other characteristics were significantly and independently prognostic in this model (Table I).

\section{Discussion}

Chronic inflammation is involved in the progression of malignant diseases and has been reported to be associated with the immune suppression that is observed in patients with advanced diseases. MDSCs, which inhibit innate and adaptive immunity, have been reported to be novel immune suppressor cells that are enhanced by inflammation and that are present in the blood circulation, lymph nodes, and tumor tissues in patients 
with cancer $(8,9)$. MDSC levels were previously measured in the PBMCs of 222 patients with esophageal, gastric, colorectal, hepatocellular, cholangiocellular, pancreatic, breast, ovarian, thyroid and lung cancer using flow cytometry (20). An additional study by another investigator provided support for the hypothesis that MDSC levels are correlated with clinical stage and metastatic disease burden in patients with breast cancer (21). Diaz-Montero et al (17) reported that the percentage of MDSCs in the total blood, as measured using flow cytometry, was increased in patients with later stage breast cancer. In that report, the average peripheral blood MSDC levels were increased with the advance of breast cancer and were highest in stage IV patients with $\geq 3$ organ systems involved in the cancer. These authors also observed that the peripheral blood MDSC levels corresponded to levels of circulating tumor cells, which are another emerging prognostic marker (17). The present study reported that the levels of MDSCs in the circulating peripheral blood were increased in patients with breast cancer compared with healthy controls. MDSC levels were significantly higher in preoperative patients than in healthy volunteers, and were decreased in postoperative patients compared with preoperative patients. In addition, MDSC levels were elevated in patients with recurrent disease to levels that were equivalent to those observed in preoperative patients. In patients with recurrent breast cancer, MDSC levels were significantly decreased following chemotherapy. The MDSC levels in preoperative patients were significantly positively correlated with IL-6 production and, conversely, were significantly negatively correlated with the production of IFN- $\gamma$ and IL-12. Th1 cells have been reported to be induced by IL-12 and to produce IFN- $\gamma$, and these cells are concerned with cellular immunocompetence (22). In addition, IL-12 is known to be a modulator of immune suppression, with significant potential as a therapeutic agent for metastatic breast cancer (23). The present study revealed that MDSC levels were inversely correlated with IL-12 production, which may suggest that MDSCs inhibited IL-12 production by dendritic cells. Furthermore, the increased production of IL-6 suggested that increased MDSC levels resulted in a Th2-dominant status. MDSC-produced IL-10 and other suppressive factors inactivate healthy dendritic cells. Increased levels of MDSCs in advanced breast cancer may therefore be involved in immune suppression in these patients through multiple immunological pathways. MDSC levels in preoperative patients were negatively correlated with a short turnover protein and with the SI, and were positively correlated with the NLR and CRP levels. These data suggested that MDSC levels in these patients in the present study were correlated not only with immune suppression, but also with malnutrition and inflammation (24). When MDSC levels were $>1.0 \%$, the prognosis of patients with stage IV breast cancer was shorter than that of other patients. However, there was no significant difference between different stages in the prognosis of patients with MDSC levels $<1.0 \%$. Thus, MDSC counts may be an important prognostic factor for patients with breast cancer. The results of multivariable analysis revealed that the risk of a poor prognosis is likely to be more dependent on MDSCs levels $>1 \%$, which were associated with a higher probability of mortality when adjusted for other potential prognostic factors. Certain anti-cancer chemotherapeutic agents, including gemcitabine, have been reported to alter MDSC levels, resulting in improvement of cellular immunocompetence (17,25). MDSC levels in the peripheral blood and in the pleural effusion of patients with metastatic breast cancer have been previously demonstrated to decrease following chemotherapy with gemcitabine (26). The results of the present study were consistent with those of previous studies. Future studies are expected to investigate novel therapeutic approaches for controlling immune suppression, malnutrition and chronic inflammation, through modulation of MDSCs by selective inhibition using molecular targeting or chemotherapy. Integrative breast cancer immunotherapies that strategically combine established therapies with breast cancer vaccines, immune checkpoint blockade or a combination of the two should result in durable clinical responses and an increased curative rate (27-29). These approaches are expected to contribute to the effective augmentation of cancer immunotherapy.

\section{References}

1. Global Burden of Disease Cancer Collaboration; Fitzmaurice C, Dicker D, Pain A, Hamavid H, Moradi-Lakeh M, MacIntyre MF, Allen C, Hansen G, Woodbrook R, et al: The global burden of cancer 2013. JAMA Oncol 1: 505-527, 2015.

2. Reeder JG and Vogel VG: Breast cancer risk management. Clin Breast Cancer 7: 833-840, 2007.

3. Tan PH and Lota AS: Interaction of current cancer treatments and the immune system: Implications for breast cancer therapeutics. Expert Opin Pharmacother 9: 2639-2660, 2008.

4. Anderson KS: Tumor vaccines for breast cancer. Cancer Invest 27: 361-368, 2009.

5. Carson WE III and Liang MI: Current immunotherapeutic strategies in breast cancer. Surg Oncol Clin N Am 16: 841-860, ix, 2007.

6. Rosenberg SA, Yang JC and Restifo NP: Cancer immunotherapy: Moving beyond current vaccines. Nat Med 10: 909-915, 2004.

7. Curigliano G, Spitaleri G, Dettori M, Locatelli M, Scarano E and Goldhirsch A: Vaccine immunotherapy in breast cancer treatment: Promising, but still early. Expert Rev Anticancer Ther 7: 1225-1241, 2007.

8. Gabrilovich DI and Nagaraj S: Myeloid-derived suppressor cells as regulators of the immune system. Nat Rev Immunol 9: $162-174,2009$.

9. Ostrand-Rosenberg S and Sinha P: Myeloid-derived suppressor cells: Linking inflammation and cancer. J Immunol 182: 4499-4506, 2009.

10. Zea AH, Rodriguez PC, Atkins MB, Hernandez C, Signoretti S, Zabaleta J, McDermott D, Quiceno D, Youmans A, O'Neill A, et al: Arginase-producing myeloid suppressor cells in renal cell carcinoma patients: A mechanism of tumor evasion. Cancer Res 65: 3044-3048, 2005.

11. Ochoa AC, Zea AH, Hernandez C and Rodriguez PC: Arginase, prostaglandins, and myeloid-derived suppressor cells in renal cell carcinoma. Clin Cancer Res 13: 721s-726s, 2007.

12. Kusmartsev S, Nefedova Y, Yoder D and Gabrilovich DI: Antigen-specific inhibition of CD8+ T cell response by immature myeloid cells in cancer is mediated by reactive oxygen species. J Immunol 172: 989-999, 2004.

13. Solito S, Falisi E, Diaz-Montero CM, Doni A, Pinton L, Rosato A, Francescato S, Basso G, Zanovello P, Onicescu G, et al: A human promyelocytic-like population is responsible for the immune suppression mediated by myeloid-derived suppressor cells. Blood 118: 2254-2265, 2011.

14. Almand B, Clark JI, Nikitina E, van Beynen J, English NR, Knight SC, Carbone DP and Gabrilovich DI: Increased production of immature myeloid cells in cancer patients: A mechanism of immunosuppression in cancer. J Immunol 166: 678-689, 2001.

15. Mosmann TR, Cherwinski H, Bond MW, Giedlin MA and Coffman RL: Two types of murine helper T cell clone. I. Definition according to profiles of lymphokine activities and secreted proteins. J Immunol 136: 2348-2357, 1986.

16. Shibata M, Nezu T, Kanou H, Abe H, Takekawa M and Fukuzawa M: Decreased production of interleukin-12 and type 2 immune responses are marked in cachectic patients with colorectal and gastric cancer. J Clin Gastroenterol 34: 416-420, 2002. 
17. Diaz-Montero CM, Salem ML, Nishimura MI, Garrett-Mayer E, Cole DJ and Montero AJ: Increased circulating myeloid-derived suppressor cells correlate with clinical cancer stage, metastatic tumor burden, and doxorubicin-cyclophosphamide chemotherapy. Cancer Immunol Immunother 58: 49-59, 2009.

18. Bevers TB, Anderson BO, Bonaccio E, Buys S, Daly MB, Dempsey PJ, Farrar WB, Fleming I, Garber JE, Harris RE, et al: NCCN clinical practice guidelines in oncology: Breast cancer screening and diagnosis. J Natl Compr Canc Netw 7: 1060-1096, 2009.

19. Le HK, Graham L, Cha E, Morales JK, Manjili MH and Bear HD: Gemcitabine directly inhibits myeloid derived suppressor cells in $\mathrm{BALB} / \mathrm{c}$ mice bearing 4T1 mammary carcinoma and augments expansion of $\mathrm{T}$ cells from tumor-bearing mice. Int Immunopharmacol 9: 900-909, 2009.

20. Gonda K, Shibata M, Nakamura I, Kenjo A, Ohtake T, Yasuda M, Suzuki S, Suzuki H, Watanabe T, Fujimori K, et al: Myeloid-derived suppressor cells in cancer patients. Gan To Kagaku Ryoho 39: 1797-1799, 2012 (In Japanese).

21. Markowitz J, Wesolowski R, Papenfuss T, Brooks TR and Carson WE III: Myeloid-derived suppressor cells in breast cancer. Breast Cancer Res Treat 140: 13-21, 2013.

22. Thakur A, Schalk D, Sarkar SH, Al-Khadimi Z, Sarkar FH and Lum LG: A Th1 cytokine-enriched microenvironment enhances tumor killing by activated $\mathrm{T}$ cells armed with bispecific antibodies and inhibits the development of myeloid-derived suppressor cells. Cancer Immunol Immunother 61: 497-509, 2012.
23. Steding CE, Wu ST, Zhang Y, Jeng MH, Elzey BD and Kao C: The role of interleukin-12 on modulating myeloid-derived suppressor cells, increasing overall survival and reducing metastasis. Immunology 133: 221-238, 2011.

24. Sinha P, Clements VK, Fulton AM and Ostrand-Rosenberg S: Prostaglandin E2 promotes tumor progression by inducing myeloid-derived suppressor cells. Cancer Res 67: 4507-4513, 2007.

25. Panis C, Lemos LG, Victorino VJ, Herrera AC, Campos FC, Colado Simão AN, Pinge-Filho P, Cecchini AL and Cecchini R: Immunological effects of taxol and adryamicin in breast cancer patients. Cancer Immunol Immunother 61: 481-488, 2012.

26. Gonda K, Shibata M, Ohtake T, Yasuda M, Abe N, Watanabe $\mathrm{K}$, Ando J, Okano M, Onozawa $\mathrm{H}$, Tachibana $\mathrm{K}$, et al: Myeloid-derived suppressor cells in patients with breast cancer. Gan To Kagaku Ryoho 39: 1363-1368, 2012 (In Japanese).

27. Diaz-Montero CM, Finke J and Montero AJ: Myeloid-derived suppressor cells in cancer: Therapeutic, predictive, and prognostic implications. Semin Oncol 41: 174-184, 2014.

28. Emens LA: Breast cancer immunobiology driving immunotherapy: Vaccines and immune checkpoint blockade. Expert Rev Anticancer Ther 12: 1597-1611, 2012.

29. Pico de Coaña Y, Masucci G, Hansson J and Kiessling R: Myeloid-derived suppressor cells and their role in CTLA-4 blockade therapy. Cancer Immunol Immunother 63: 977-983, 2014. 